\title{
WHISTLERS »ARRANGEMENT IN GREY AND BLACK: PORTRAIT OF THE PAINTER'S MOTHER«. MEISTERWERK ZWISCHEN KARRIERE UND KRITIK
}

\author{
Yes - one does like to make one's mummy just as nice \\ as possible.
}

James MCNeill Whistler im Gespräch Mit Harper
PenNington ${ }^{1}$

Als im Spätsommer 1871 Maggie Graham, die fünfzehnjährige
Tochter des britischen Parlamentsmitglieds William Graham,
nicht zur verabredeten Porträtsitzung in Chelsea erschien, suchte der Künstler ein Ersatzmotiv und bat seine im gleichen Haus lebende Mutter, für ihn Modell zu stehen. ${ }^{2}$ Eine Kaltnadelradierung, von der nur ein einziger Abzug bekannt ist, bevor James McNeill Whistler (1832-1903) die Kupferplatte durch Zerkratzen entwertete (Abb. 1, G.103 ${ }^{3}$ ), mag von der ursprünglich vorgesehenen Pose eine Ahnung vermitteln. ${ }^{4}$ Das lange Modellstehen wur-
1 Joseph und Elizabeth Pennell, The Life of James McNeill Whistler, 2 Bde., London 1908, Bd. 1, 170. 2 Anna Matilda Whistler an ihre Schwester Catherine Jane Palmer, 3.-4. November 1871, in: Margaret F. MacDonald, Patricia de Montfort und Nigel Thorp (Hrsg.), The Correspondence of James McNeill Whistler, 1855-1903, und Georgia Toutziari (Hrsg.), The Correspondence of Anna McNeill Whistler, 1855-1880, Online-Ausgabe, University of Glasgow 2003-2010, URL: http://www.whistler.arts.gla.ac.uk/correspondence [letzter Zugriff am 21.07.2014] [im Folgenden: GUW], Nr. 10071.

3 G kürzt das neue Werkverzeichnis der Radierungen Whistlers ab, Margaret F. MacDonald, Grischka Petri, Meg Hausberg, Joanna Meacock (Hrsg.), James McNeill Whistler: The Etchings, a catalogue raisonné, Online-Ausgabe, University of Glasgow, 2011, URL: http:// etchings.arts.gla.ac.uk [letzter Zugriff am 21.07.2014], das Edward G. Kennedy, The Etched Work of Whistler, New York 1910, als Referenz ablöst.

4 Joseph und Elizabeth Pennell (wie Anm. 1), Bd. 1, 215, behaupten, die Radierung habe mit dem Gemälde nichts zu tun, doch hält sie eine mögliche aufrechte Pose des geplanten Porträts fest. Dennoch bleibt die Datierung der Grafik vage; vgl. Margaret F. MacDonald, 


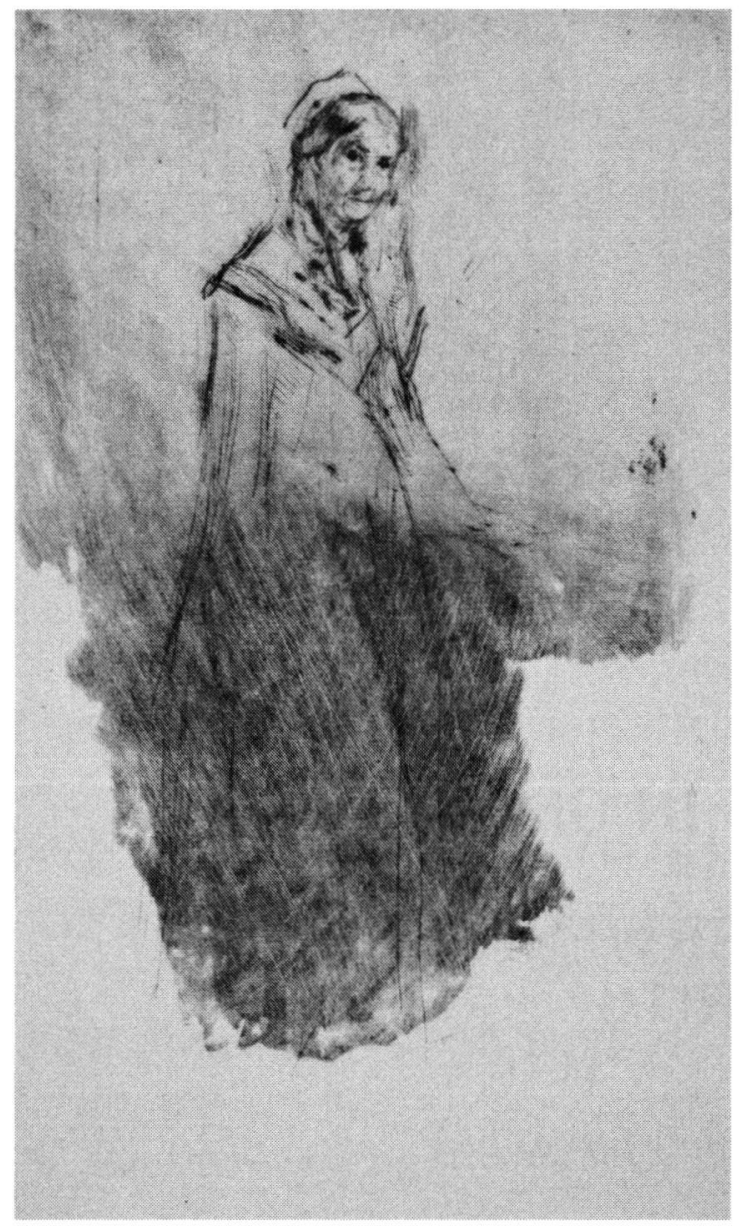

ABB. 1: JAMES MCNEILL WHISTLER, WHISTLER'S MOTHER (G.103), UM 1871, RADIERUNG, $252 \times 153 \mathrm{MM}$, WASHINGTON, FREER GALLERY OF ART (F.1903.252)

de der 67jährigen indes bald zu anstrengend. ${ }^{5}$ Whistler setzte seine Mutter auf einen Stuhl. Für die neue Komposition wählte er die Rückseite einer begonnenen, aber nicht zu Ende gebrachten Leinwand mit den Maßen 144,3×162,5 cm aus. ${ }^{6}$ Im Oktober war das neue Gemälde vollendet.

Arrangement in Grey and Black: Portrait of the Painter's Mother (Abb. 2, YMSM.101') zeigt im scharfen Linksprofil die auf ei-

The Painting of Whistler's Mother, in: dies. (Hrsg.), Whistler's Mother: An American Icon, Aldershot 2003, 29-63, 34. Lange wurde anhand einer aus Indizien geschlossenen Datierung eines Briefes an Henri FantinLatour angenommen, dass Whistler bereits 1867 an einem Porträt seiner Mutter gearbeitet habe; vgl. John Sandberg, >Japonisme< and Whistler, in: Burlington Magazine 106, 1964, 500-507, 507. Inzwischen konnte dieser Brief Whistlers, in dem er erwähnt, er wolle sein neues Werk fotografieren lassen, überzeugend auf August 1872 datiert werden (GUW 08041).

5 "/ so interested stood as a statue! but realized it to be too great an effort « - Anna Matilda Whistler an ihre Schwester Catherine Jane Palmer, 3.-4. November 1871, GUW 10071.

6 Die letzte Restauratorin des Gemäldes, Sarah Walden, bestätigt, dass die Leinwand schon einmal verwendet worden war, doch verraten die Röntgenbilder kein konkretes Motiv; siehe Sarah Walden, Whistler and His Mother: An Unexpected Relationship, London 2003 , 53-54. Das Gemälde wurde 1891 mit einer weiteren Leinwand unterlegt (»doubliert«), so dass das einmal begonnene Gemälde nicht mehr mit bloßem Auge erkennbar ist. Während dieser Vorgang für gewöhnlich eine Leinwand stabilisieren soll, könnte Whistler vor allem beabsichtigt haben, das alte Gemälde verschwinden zu lassen, als Arrangement in Grey and Black vom französischen Staat angekauft wurde (Walden, a.a.O., 204).

7 YMSM kürzt das Werkverzeichnis der Gemälde Whistlers ab: Andrew McLaren Young, Margaret F. MacDonald, Robin Spencer, Hamish Miles, The Paintings of James McNeill Whistler, New Haven 1980. 


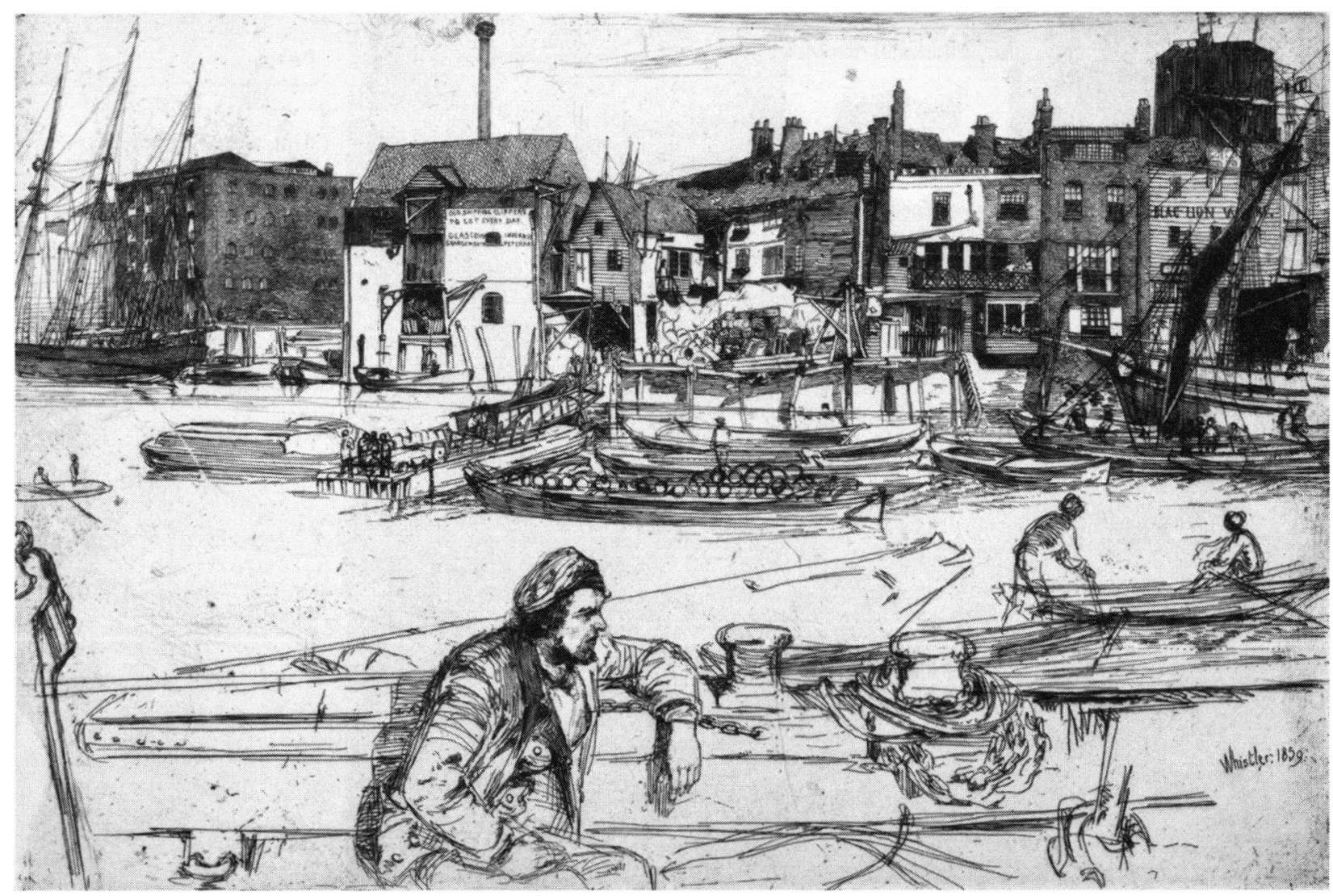

ABB. 3: JAMES MCNEILL WHISTLER, BLACK LION WHARF (G.54), 1859, RADIERUNG, $152 \times 225$ MM,

GLASGOW, THE HUNTERIAN (46783)

I.

\section{KüNSTLERISCHE TECHNIK UND MATERIELLE}

\section{VER ÄNDERLICHKEIT}

Es gibt von Walter Greaves (1846-1930) mehrere Gemälde, die Whistler darstellen, wie er Arrangement in Grey and Black malt (Abb. 4), die allerdings aus der Erinnerung entstanden und dokumentarischen Fragen zur Entstehung weder dienen können noch sollten. ${ }^{9}$ Wir sind auf den Befund des Werkes selbst angewiesen. Mehrere Pentimenti (noch erkennbare malerische Korrekturen) sowie die Untersuchung des Bildes unter (stark von der Seite kommendem) Streiflicht, Infrarotlicht und Röntgenstrahlen haben verraten, dass Whistler ursprünglich die Figur mehrere Zentimeter höher positioniert und mit etwas weiter nach unten hängendem Arm konzipiert hatte. Das Kleid nahm mehr Raum ein, war aber nicht bis zum unteren Bildrand gezogen. Der Bilderrahmen und der rechte Kimonorand wanderten etwas weiter nach links, und ganz rechts fügte Whistler noch einen schmalen Rand eines weiteren Bilderrahmens ein, nachdem das Bild bereits getrocknet war. ${ }^{10}$

Das Gemälde hängt heute im Pariser Musée d'Orsay. Sarah Walden, die das Gemälde Ende der 1980er Jahre restaurierte, be-
9 Greaves wurde nach Whistlers Tod als dessen »Schüler« und Zeitzeuge entdeckt. Seine Erinnerung war indes nicht immer akkurat, insbesondere in Bezug auf verschiedene Datierungen. Die privat gemalten Bilder Greaves' blieben dem Kunstpublikum die längste Zeit verborgen. Sie feierten später einen kurzen Erfolg in London, als die Kunsthandlung Goupil 1911 eine Ausstellung organisierte. Zu Greaves siehe Tom Pocock, Chelsea Reach: The Brutal Friendship of Whistler and Walter Greaves, London 1970.

10 MacDonald (wie Anm. 8), 79 mit einer instruktiven Grafik der Änderungen; MacDonald (wie Anm. 4), 54. 


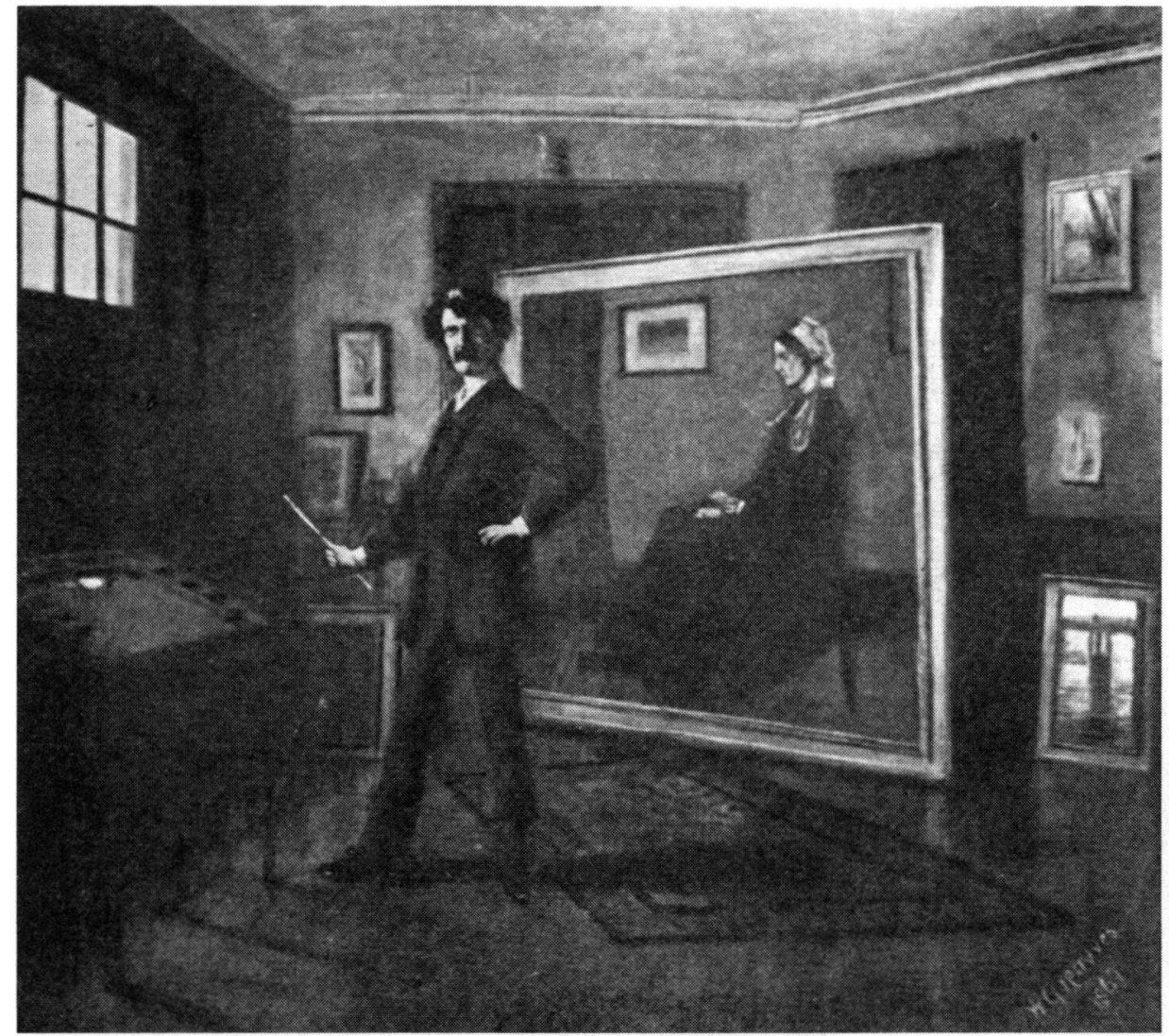

tont, dass der heutige relativ matte Eindruck des Porträts ein anderer als zu seiner Entstehungszeit ist. Beispielsweise ist die ursprünglich durch das Schwarz des Kleides durchscheinende Linie der Wandpaneele längst nicht mehr erkennbar. ${ }^{11}$ Dies hängt mit den von Whistler verwendeten Materialien zusammen. Walden fasst die drei Hauptbestandteile der Wirkung des Gemäldes so zusammen: graues Licht, raue Leinwand und dünne Farbe. ${ }^{12}$ Bereits Greaves erinnerte sich, dass Whistler für die Mutter stark mit Terpentin verdünnte Farbe - die von ihm so genannte "Sauce« - verwendet hatte. ${ }^{13}$ Der dünne, trocken wirkende Farbauftrag betonte die Leinwandstruktur, die als eigentliche Rückseite besonders rau wirkte. Whistler setzte diese Textur sehr effektvoll ein, vor allem für die Darstellung der weißen Textilien von Ärmelenden, Haube und Taschentuch, das Greaves als "nichts als ein Bisschen Weiß und Öl« beschrieb. ${ }^{14}$ Diese verdünnte Farbe wurde allerdings von der rauen Leinwandfaser absorbiert und verlor dadurch schnell an Glanz. Möglicherweise mischte Whistler sie mit einer geléeartigen Firnis aus Mastix (ein Harz aus griechischen Pistazienbäumen) und Öl, um diesem Glanzverlust vorzubeugen. ${ }^{15}$ Gleichzeitig verwendete er sehr feine Pigmente. Frisch gemalt, schließt Walden, müssen die schwarzgrauen Partien einmal den Glanz von ja-
Petri

ABB. 4: WALTER GREAVES, INTERIOR OF THE LINDSEY ROW STUDIO: WHISTLER PAINTING THE PORTRAIT OF HIS MOTHER, UNZUTREFFEND DATIERT »1868«, ÖL AUF LEINWAND, FORMAT UND VERBLEIB UNBEKANNT

11 Walden (wie Anm. 6), 219. 12 Walden (wie Anm. 6), 182, 195.

13 Elizabeth R., Joseph Pennell, The Whistler Journal, Philadelphia 1921, 116; auch Thomas Way, Memories of Whistler, London 1912, 69-70, beschreibt Whistlers Verwendung von stark mit Terpentin verdünnter Farbe. 14 Pennell (wie Anm. 1), Bd. 1, 168. Neben Walter stand auch dessen Bruder Henry (1844-1904) Whistler in den 186oer und 1870er Jahren mit praktischen Hilfen zur Seite.

15 Walden (wie Anm. 6), 184. 


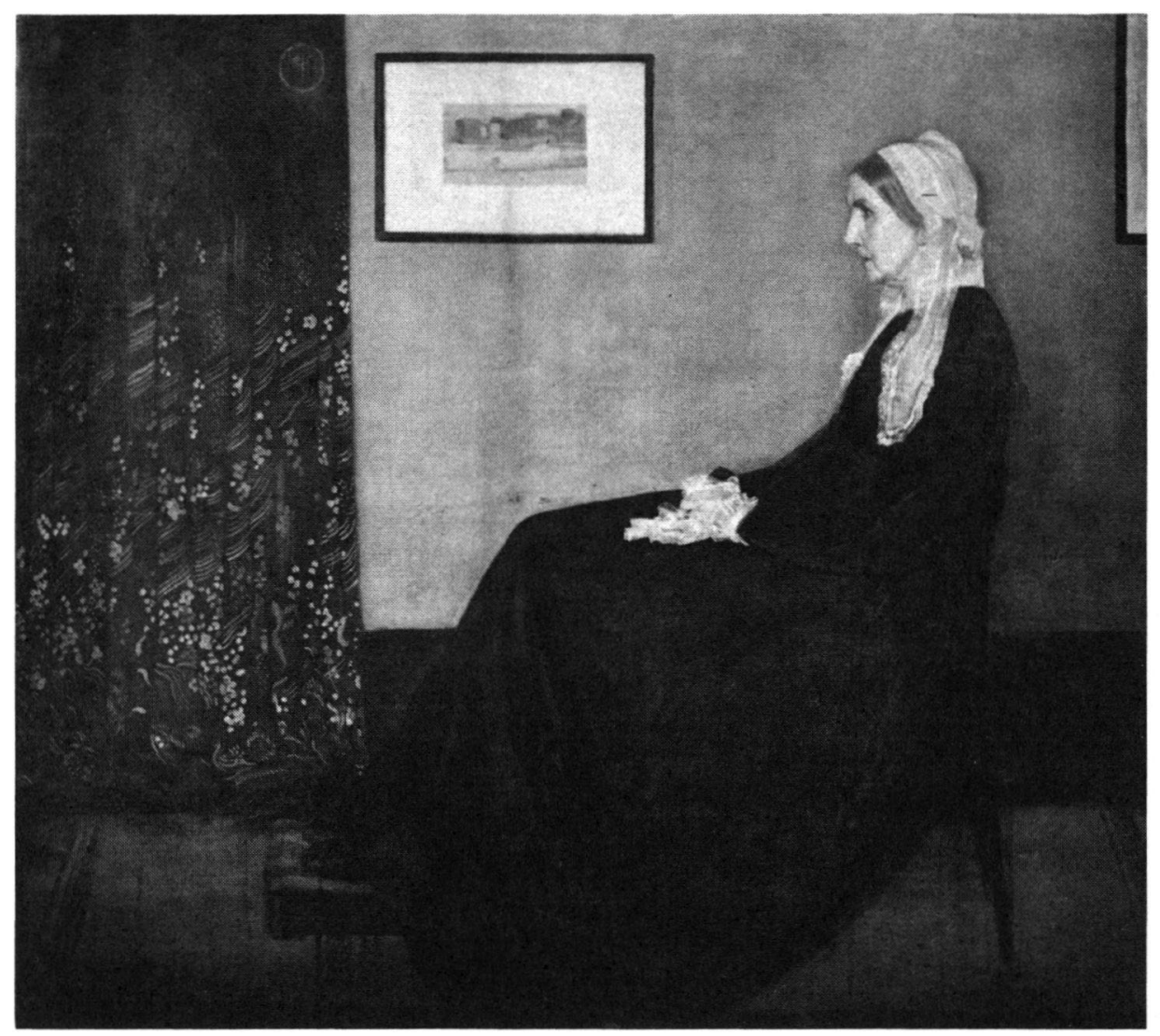

ABB. 5: ARRANGEMENT IN GREY AND BLACK: PORTRAIT OF THE PAINTER'S MOTHER, FOTOGRAFIE VON JOHN ROBERT PARSONS, 1872

panischer Tusche mit der Transparenz des Öls verbunden haben. Schnell vergrauten sie aber durch das Einsinken der Farbe in die Leinwand. Der spektakuläre Farbkontrast ließ nach. Whistler versuchte wohl, diesem Vorgang mit neuen Firnisschichten aus Mastix oder Kopal entgegenzuwirken. Dies führte über die Zeit jedoch zu Fleckenbildung, weil die Leinwand die neuen Schichten unregelmäßig aufnahm. Außerdem vergilbten die Harze. All dies verstopfte die offene Leinwandstruktur. Durch das Doublieren der Leinwand 1891 wurde Klebstoff in die noch nicht voll ausgehärteten Farb- und Firnisschichten gepresst. ${ }^{16}$ Für die Restaurierung bedeutete dies, dass ein ursprünglicher, firnisloser Zustand nicht wieder hergestellt werden konnte. ${ }^{17}$ Ein Vergleich heutiger Aufnahmen (Abb. 2) mit einer Photographie John Robert Parsons' von 1872 (Abb. 5) zeigt einerseits die ursprünglich höhere Transparenz der dunklen Farben im Bild, stellt aber andererseits die Frage nach den Veränderungen, welche die Photographie des Gemäldes durchlaufen hat.

Bereits die für Arrangement in Grey and Black verwendeten Materialien und seine genaue technische Untersuchung geben Hinweise auf Whistlers ästhetische Absichten. Sie dokumentieren aber auch - obwohl das Gemälde mit seinen 140 Jahren ver-

16 Walden (wie Anm. 6), 200-204.

17 Walden (wie Anm. 6), 215. Sie schildert im Folgenden interessante Einzelheiten ihrer Restaurierung. 


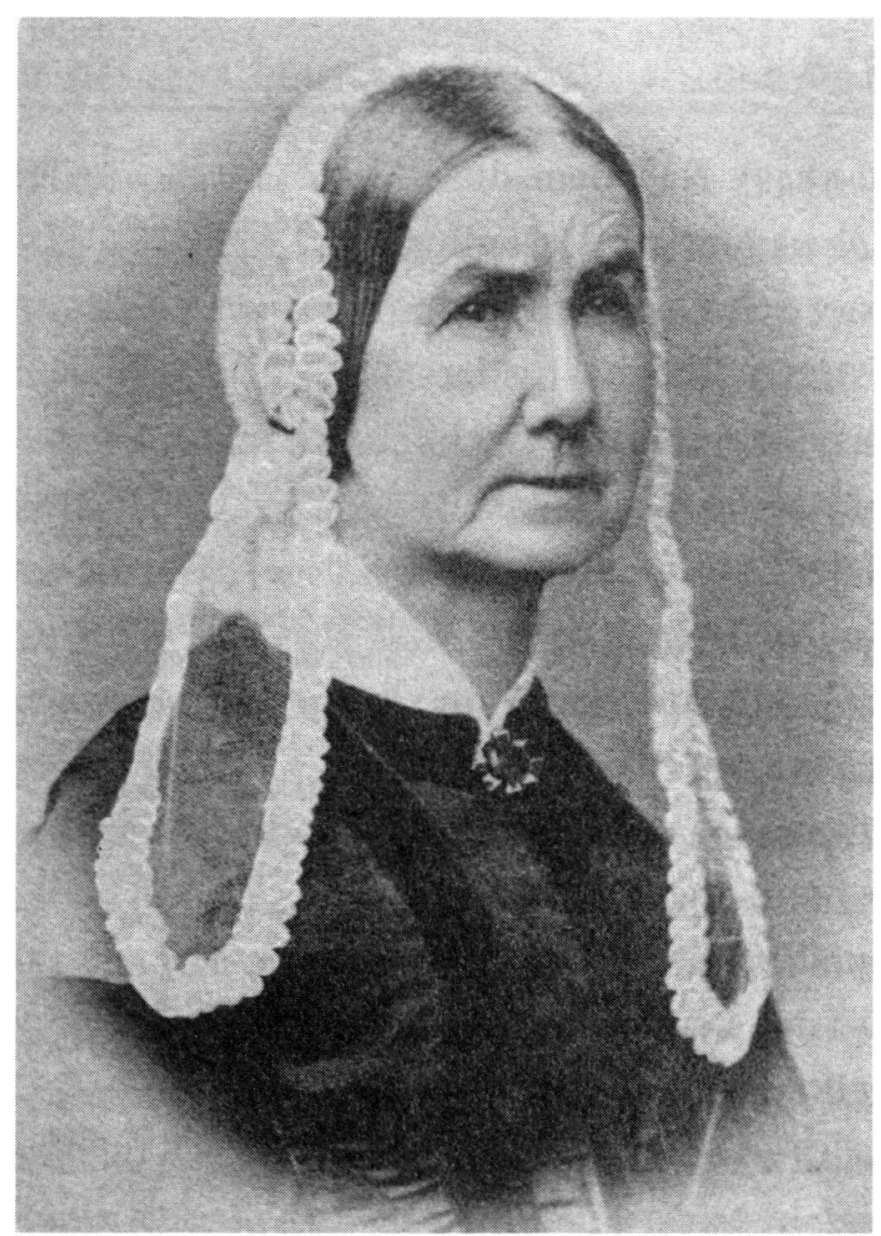

ABB. 6: ANNA MATILDA

MCNEILL WHISTLER, UM 1870, FOTOGRAFIE

gleichsweise jung ist - das Problem der materiellen Veränderlichkeit von Kunstwerken. Die Untersuchungsmethoden haben sich seit der Restaurierung des Gemäldes in den 1980er Jahren weiter entwickelt, was nicht zuletzt zum Aufschwung der »technischen Kunstgeschichte« geführt hat. Diese versucht auch außerhalb skandalträchtiger Fälschungsfälle, über naturwissenschaftliche Ansätze und Methoden zu Erkenntnissen zu gelangen, wie sie für Whistlers Porträt seiner Mutter bereits ansatzweise vorliegen. Dieser methodische Weg ist allerdings noch nicht zu Ende gegangen.

II.

\section{SUJET UND BIOGRAPHIE}

Die Bedeutung eines Porträts liegt jedenfalls zum Teil in der dargestellten Person begründet. Der Hinweis auf »the painter's mother« im Bildtitel identifiziert die dargestellte Person, und der Vergleich mit einer zeitgenössischen Photographie (Abb. 6) bestätigt dies. Wer war Anna Matilda Whistler? Das Arrangement in Grey and Black erlaubt verschiedene Rückschlüsse. Der Ehering verweist auf Whistlers Vater, den amerikanischen Eisenbahninge- 
nieur George Washington Whistler (1800-1849). Nachdem dieser 1827 Witwer wurde, heiratete er 1831 Anna Matilda McNeill. Der zukünftige Künstler James war ihr erster Sohn, von den weiteren Kindern überlebte nur der spätere Arzt William Whistler (1836-1900). Der russische Zar berief Annas Ehemann 1842 nach St. Petersburg, um den Bau der Eisenbahnstrecke nach Moskau zu leiten. Dort starb der Ingenieur 1849 an der Cholera; die Familie kehrte nach Aufenthalten in England in ihre Heimat der USA zurück. ${ }^{18}$ Sechs Jahre später wanderte der junge James aus, um sich in Paris als Künstler niederzulassen; 1859 zog er nach London um. 1863, während des Sezessionskrieges, zog seine Mutter bei ihm ein. Anna Matildas Anwesenheit hatte angenehme wie weniger angenehme Auswirkungen. Sie stellte einen Störfaktor in Whistlers Beziehung zu Joanna Hiffernan dar, half ihrem Sohn aber auch bei der Organisation von Ausstellungen und dem Vertrieb seiner Radierungen. 1875 zog sie nach Hastings (East Sussex), wo sie 1881 starb. Die familiäre Beziehung ist allerdings nicht das vordergründige Thema des Bildes. Whistler selbst ist nur stellvertretend durch seine Radierung (Abb. 3) und durch das von ihm gestaltete Interieur seines Ateliers in der No. 2 Lindsey Row in Chelsea präsent. ${ }^{19}$ Dass Anna Matilda seine Mutter ist, geht nur aus dem Titel hervor. Demgegenüber zeichnen sie das schwarze Gewand und der Ehering als Witwe aus, ähnlich den Selbstdarstellungen Königin Victorias nach dem Tod ihres Prinzgemahls Albert 1863. Die Farben aus dem Arrangement in Grey and Black stehen damit nicht nur für sich, sondern auch für Alter und Trauer.

Ein Gemälde, das eine bestimmte Person ins Bild setzt, gibt also zunächst Anlass zu historisch-kontextualisierenden Fragestellungen zu jener Person. Wir erfahren in diesem konkreten Fall etwas über Anna Matilda Whistler selbst, über ihre Familie und je nach Tiefe der Recherche - ihre Haushaltsführung, ${ }^{20}$ die Internationalisierung des Eisenbahningenieurwesens, Krankheiten und Konventionen der Witwenschaft sowie der Mode. Dennoch erschöpft sich das Porträt nicht in der biographischen Interpretation der dargestellten Person.
18 Eine ausführliche Auseinandersetzung mit Anna McNeill Whistler hat Georgia Toutziari geführt. Die von ihr edierte Korrespondenz ist Teil der Whistler Correspondence (GUW, wie Anm. 1); siehe ferner dies., Anna Matilda Whistler: A Life, in MacDonald (wie Anm.4), 13-26; Ambitions, Hopes, and Disappointments: The Relationship of Whistler and His Mother as Seen in Their Correspondence, in: Lee Glazer et al. (Hrsg.), James McNeill Whistler in Context, Washington DC 2008,

205-214.

19 Vgl. zu Whistlers Aktivitäten als Innenarchitekt und Dekorateur Deanna Marohn Bendix, Diabolical Designs: Paintings, Interiors, and Exhibitions of James McNeill Whistler, Washington/ DC 1995, zu den Räumen in No. 2 Lindsey Row (heute 96 Cheyne Walk) 86-98. Die grauen Wände des Ateliers sind auch in weiteren Porträts Whistlers zu sehen wie Arrangement in Grey and Black, No. 2: Portrait of Thomas Carlyle (YMSM.137) und Harmony in Grey and Green: Portrait of Miss Cicely Alexander (YMSM.129).

20 Siehe Margaret F. MacDonald (Hrsg.), Whistler's Mother's Cook Book, 2. Aufl., San Francisco 1995. 
III.

\section{DAS GEMÄlde in DER SOMmERAUSSTELlung DER ROYAL}

ACADEMY 1872. LEGENDENBILDUNG UND SELBSTSTILISIERUNG

Nachdem das Gemälde vollendet war, brachte Whistler es auf den Landsitz seines Hauptsammlers Frederick R. Leyland nach Speke Hall bei Liverpool. Hier wurde es probeweise zwischen Whistlers neues Porträt Leylands und einen Velázquez gehängt und behauptete sich, wie Whistler seiner Mutter gegenüber bemerkte, gegenüber beiden sehr gut. ${ }^{21}$ Auch Künstlerkollegen äußerten sich positiv über das Porträt. Ford Madox Brown hielt es für »good and beautiful«;22 Dante Gabriel Rossetti schrieb, es müsse Whistler ein Leben lang glücklich machen. ${ }^{23}$ Whistler reichte es für die jährliche Sommerausstellung der Royal Academy 1872 ein. Dort wurde es schließlich unter der Nummer 941 ausgestellt.

Zwischen Einreichung und Ausstellung muss es allerdings innerhalb der Royal Academy Streitigkeiten über das Bild gegeben haben. Brown berichtete von Gerüchten, das Bild sei abgelehnt worden, ${ }^{24}$ Whistler sechs Jahre später hingegen in einem Interview davon, dass erst William Boxalls Drohung, seine Akademiemitgliedschaft niederzulegen, die Jury dazu bewog, es auszustellen. ${ }^{25}$ In der Erinnerung des in London ansässigen amerikanischen Künstlers George H. Boughton (1833-1905) waren die Ereignisse allerdings etwas weniger dramatisch. Danach habe sich Boxall gegen die Idee verwahrt, das Gemälde etwas höher an der Wand zu positionieren, um darunter kleinformatige Bilder anzubringen, für die der Platz knapp war. ${ }^{26}$ Whistler schließlich sei erfreut gewesen, dass sein Werk einen guten Platz »on the line «, also in einer den Betrachtern gut zugänglichen Höhe, erhalten hatte. ${ }^{27}$

Worüber genau in der Royal Academy im Frühjahr 1872 gestritten wurde, wissen wir nicht, und werden wir wohl auch nie erfahren. Die Geschichte der Ablehnung des Porträts der Mutter, ein Meisterwerk, durch die Royal Academy, wurde aber in anderen Magazinen aufgenommen und weiter getragen: 1887 durch Whistlers Galeristen Walter Dowdeswell, vom Künstler beraten, in einem Artikel für das angesehene Londoner Art Journal, ${ }^{28} 1908$

21 Anna Matilda Whistler an ihre Schwester Catherine Jane Palmer, 3.-4. November 1871, GUW 10071. Auf der Rückfahrt nach London mit der Bahn brach ein Feuer im Zug aus, doch überstand das Gemälde die Fahrt unversehrt; Anna Matilda Whistler an James H. Gamble, 10.-20. April 1872, GUW 06549.

22 Ford Madox Brown an George Rae, n.d. [Frühjahr 1872], in: Ford Madox Hueffer [= Ford Madox Ford], Ford Madox Brown: A Record of His Life and Work, London 1896,280

23 Dante Gabriel Rossetti an James McNeill Whistler, [Oktober / November] 1872, GUW 09415.

24 Ford Madox Brown an George Rae (wie Anm. 22). 25 Celebrities at Home, No. XCII: Mr. James Whistler at Cheyne Walk, The World, 22. Mai 1878, 4-5, 4. $26 \mathrm{G}$ [eorge] $\mathrm{H}$. Boughton, A Few of the Various Whistlers I Have Known, in: The Studio, 30, 1903-04, 208-218, 215.

27 Verschiedene Autoren geben die Höhe der »Linie«, die ursprünglich durch eine Leiste angegeben war, etwa zwischen 2, 20 und 2, $40 \mathrm{~m}$ an. Diese Linie stellte den oberen Rand des an die Wand anzubringenden Gemäldes dar. Daraus können mit Hilfe der Augenhöhe auch Rückschlüsse über die ideale Größe eines solchen Akademiegemäldes gezogen werden. Vgl. George Dunlop Leslie, The Inner Life of the Royal Academy, London, 1914, 75; Report of the Commissioners Appointed to Inquire into the Present 
durch die noch von ihm selbst autorisierten Biographie Whistlers von Elizabeth R. und Joseph Pennell, ${ }^{29}$ und schließlich im Werkverzeichnis der Gemälde. Das einstmalige Gerücht war schnell historische Tatsache geworden und sorgte für eine herausgehobene Stellung des Werks in der Biographie und in der Karriere Whistlers - mit seiner eigenen nachhaltigen Unterstützung. Denn die treibende erzählerische Kraft der Anekdote der Ablehnung war Whistler selbst. In seiner drastischeren Version konnte er sich als vom Establishment abgelehnten Außenseiter darstellen. Das sechs Jahre später gegebene Interview in The World von 1878 war Teil der Pressearbeit, die Whistler in Vorbereitung seines Prozesses gegen den Kunstkritiker John Ruskin leistete. ${ }^{30}$ Whistler hatte begonnen, sich als unverstandenes Genie darzustellen; 1872 war ihm aber an einem Erfolg an der Royal Academy noch viel gelegen. Er war bis dato nie ein anti-akademischer Maler gewesen, sondern erstrebte sogar seine Aufnahme in den illustren Kreis. ${ }^{31}$ Tatsächlich wurde von Whistlers siebzehn zwischen 1860 und 1872 für die Sommerausstellungen eingereichten Gemälden nur das White Girl (YMSM.38) im Jahre 1862 abgelehnt. Weitaus öfter schaffte es der Künstler schlicht nicht, seine Werke rechtzeitig zur Sommerausstellung einzureichen, wie 1868 und 1869, als er nichts ausstellte, oder 1870, als er statt der nie vollendeten Three Girls sein fünf Jahre altes Gemälde The Balcony (YMSM.56) zeigte. Whistlers Mutter erinnert uns daran, dass Künstleraussagen oft einen weiteren Zweck verfolgen als nur die Fakten wiederzugeben.

IV.

\section{Arrangement oder Porträt, Form versus Inhalt?}

Whistler erreichte 1871 mit dem Porträt seiner Mutter, woran er sich in den Jahren zuvor vergeblich abgearbeitet hatte. Die von ihm im Interview erwähnten »Verdienste als Arrangement« wie auch der von ihm als Bestandteil des Bildtitels etablierte Begriff des Arrangements verweisen auf rein ästhetische Qualitäten. In den 1860er Jahren hatte Whistler mehrere Anläufe unternommen, eine dem modernen Ästhetizismus entsprechende, das Schöne betonende Bildsprache zu entwickeln. Hierzu gehörten seine White Girls, die später so betitelten Symphonies in White,
29 Pennell (wie Anm. 1), Bd. 1, 157.

30 Vgl. Linda Merrill, A Pot of Paint: Aesthetics on Trial in Whistler v. Ruskin, Washington, DC 1993, 68. 31 In einem erhaltenen, undatierten Briefentwurf an die Royal Academy (GUW 05258) beschwert sich Whistler, dass sein Name von der Kandidatenliste gestrichen worden sei. 
die er 1862,1864 und $1865-67$ vollendete. ${ }^{32}$ Die seinerzeit wohl erfolgreichste Formel verfolgte Whistler mit Darstellungen junger Frauen inmitten fernöstlicher Kunstgegenstände, etwa in The Golden Screen (YMSM.6o), das Joanna Hiffernan in einem Kimono inmitten japanischer Holzschnitte vor einem japanischen Wandschirm zeigt, und The Lange Lijzen of the Six Marks (YMSM.47), in dem das sitzende Modell inmitten zahlreicher blau-weißer Porzellangefäße eine Vase in den Händen hält. Der Kunsthistoriker John Sandberg sah Whistlers Porträt seiner Mutter als vereinfachte, rationalisierte Variante dieses Gemäldes. ${ }^{33}$ Anstatt einen nach Verkleidung aussehenden Japonismus der Inszenierung des exotischen Objekts umzusetzen, wie noch in den 186oer Jahren, wandte Whistler nunmehr japanische ästhetische Prinzipien unmittelbar an, die er aus den mittlerweile stark verbreiteten Holzschnitten kannte: eine flächige Komposition mit einer reduzierten Farbskala und einer zurückgenommenen Räumlichkeit. Die erwähnte Anspielung auf japanische Materialien durch die wie japanische Tusche wirkende schwarze Farbe unterstützte diesen Effekt. Mit Arrangement in Grey and Blackgelang Whistler damit in der Gattung des Porträts, was ihm zuvor nicht hatte gelingen wollen: eine im Bild autonome, aber handlungslose Figur, eine Person als ästhetisches Arrangement, anscheinend mühelos und elegant komponiert, ein perfektes Beispiel für den Ästhetizismus des "Art for Art's Sake«, denn durch den privaten Charakter erhob das Gemälde nicht einmal einen kommerziellen Anspruch. ${ }^{34}$

Whistler war die Qualität seines Gemäldes sehr bewusst. Im erwähnten Interview mit The World äußerte er sich auch zu seinen künstlerischen Ideen, und dem Porträt seiner Mutter kam dabei eine Schlüsselstellung zu. Der Eckstein seiner Kunstphilosophie sei, die Malerei, gleich der Musik für den Klang, als Poesie des Sehens zu begreifen. Der Inhalt habe indes nichts mit Klang- oder Farbharmonien zu tun. Kunst solle von jeglichem Geschwätz unabhängig sein, für sich stehen und den künstlerischen Sinnen von Auge oder Ohr allein gefallen, ohne dies mit ihnen vollkommen fremden Gefühlen zu verwechseln wie Andacht, Mitleid, Liebe, Vaterlandsliebe oder Ähnlichem. Diese Gefühle hätten mit Kunst
32 Symphony in White, No. 1: The White Girl, National Gallery of Art, Washington DC (YMSM.38); Symphony in White, No. 2: The Little White Girl, Tate Britain, London (YMSM.52); Symphony in White, No. 3, Barber Institute of Fine Arts, Birmingham (YMSM.61). 33 Sandberg (wie Anm. 4), 507.

34 Grischka Petri, Arrangement in Business. The Art Markets and the Career of James McNeill Whistler, Hildesheim 2011, 140. 
nichts zu tun, weshalb Whistler darauf bestand, seine Werke Arrangements und Harmonien zu nennen:

»Dort, gegenüber meinem Bett, ist das Bild [...] meiner Mutter, in der Royal Academy ausgestellt als sArrangement in Grau und Schwarz. Nun, das ist, was es ist. Für mich ist es als ein Bild meiner Mutter interessant, aber was kann oder sollte das Publikum für ein Interesse an der Identität des Porträts haben? Es muss anhand seiner Verdienste als >Arrangement stehen oder fallen [...]. « $^{35}$

Die Kritiker der Sommerausstellung der Royal Academy 1872 erkannten diese Verdienste in der Regel, teilten jedoch nicht immer die Annahme, dass derart inhaltslose, sich selbst genügende formale Qualitäten ein gutes Kunstwerk ausmachten. So befand der Rezensent der Birmingham Daily Post das Gemälde für gewitzt, aber unattraktiv - er bevorzugte Edward Poynters junge wie attraktive Protagonistin aus Perseus und Andromeda (Nr. 505). ${ }^{36}$ Der Examiner beurteilte es als "erstaunlich", wie viel Whistler mit zwei Farben erreicht habe, auch wenn kein wirklicher Zweck einer solchen Beschränkung erkennbar sei. ${ }^{37}$ Die Pall Mall Gazette schrieb, das Porträt sei bewunderungswürdig für seine feinsinnigen Farbtöne und seine würdige Empfindung des Daseins einer alten Dame ${ }^{38}$ - ein Sowohl-als-auch-Lob, das zwar äußerst knapp gehalten war, aber doch Form wie Inhalt des Gemäldes berücksichtigte. Der Kritiker der Times hingegen empfand die beschränkte Farbpalette als angenehm, beanstandete aber die fehlende Festigkeit des Kopfes und die Aufdringlichkeit der weißen Farbe der Radierung an der Wand ${ }^{39}$ Hier wurden also malerischästhetische Mängel moniert und Whistler vorgeworfen, die erstrebte Farbharmonie verfehlt zu haben. Der Standard wiederum befand, Whistlers Leinwand sei groß, und größtenteils leer. Die gedämpften Grautöne von Boden und Wand würden bei jedem anderen Maler monoton wirken, und in dieser erhabenen Kammer sitze die Dame im Trauergewand. Das Porträt sei aber zu streng und deshalb bei den Ausstellungsbesuchern nicht beliebt - der Künstler hätte außerdem ein paar Details hinzufügen können, die das Bild interessanter machten. ${ }^{40}$
35 »There, hanging opposite my bed, is the picture of [...] my mother, exhibited at the Royal Academy as an ,Arrangement in Grey and Black.< Now that is what it is. To me it is interesting as a picture of my mother; but what can or ought the public to care about the identity of the portrait? It must stand or falton its merits as an sarrangement; [...].« Celebrities at Home, No. XCII: Mr. James Whistler at Cheyne Walk, The World, 22. Mai 1878, 4-5, 4. Leicht gekürzt nahm Whistler diese Stelle auch in seinem Buch The Gentle Art of Making Enemies, London 1890,

2. Aufl. 1892, 128, auf.

36 At the Academy, Birmingham Daily Post, 14. Mai 1872, 6 .

37 The Royal Academy, The Examiner, 22. Juni 1872 621-622, 622. Zu einem ähnlichen Urteil kam der Verfasser der Zeitung The Graphic vom gleichen Tag (Royal Academy, The Graphic, 22. Juni, 590). 38 The Royal Academy, Pall Mall Gazette, 3. Mai 1872, 10-11, 11.

39 Exhibition of the Royal Academy, The Times, 21. Mai $1872,7$.

40 The Exhibition of the Royal Academy, The Standard, 22. Mai 1872, 6. Whistler notierte später ironisch, geeignete Gegenstände seien eine Bibel und ein Glas Sherry; Notiz von Walter Sickert neben dem Zeitungsausschnitt aus Whistlers Sammlung, Glasgow University Library, Special Collections, PC.1, fol. 9. 
Der später in Whistlers Interview angesprochene Gegensatz der Interessen an den formalen Qualitäten des Porträts und den Sympathien, die der alten Dame entgegengebracht wurden, zeichnet sich schon in diesen ersten Kritiken an Arrangement in Grey and Black: Portrait of the Painter's Mother ab. Freilich kann trotz aller Vollendung in Form und Farbe nicht davon abgesehen werden, dass das Porträt eben eines ist und somit nicht ohne Inhalt. In seiner Rezension der gedruckten Ausgabe von Whistlers Ten O'Clock Lecture $^{41}$ merkte der Poet Algernon Swinburne (1837-1909) ironisch an, auch Whistlers Porträts seien nicht nur Arrangements, sondern wirkten auch auf Verstand und Gefühl ein. Der Künstler könne nur schwerlich behaupten, dass er nie beabsichtigt habe, "pathosvolle Bedeutung und zarte Tiefe des Ausdrucks« in das Porträt seiner Mutter zu legen: »Die skandalöse Tatsache bleibt, dass er es getan hat «. ${ }^{42}$ Whistlers Betonung der inhaltsfernen Eigenschaften dieses Gemäldes wie eigentlich seines Gesamtwerkes integriert ihn in eine Tradition der Moderne, die auf der Geschichte der Abstraktion aufbaut, gewissermaßen in einer Geschichte vom zweiten Teil von Whistlers Bildtitel hin zum ersten. Dabei kann es an dieser Stelle nur bei skizzenhaften Andeutungen bleiben. Whistlers Malerei wurde seit den 189oer Jahren von einer jüngeren Generation von britischen Kunstkritikern des »New Art Criticism« positiv gewürdigt, etwa Dugald S. MacColl (1859-1948) und Robert A. M. Stevenson (1847-1900). Diese bewerteten eine gelungene Bilderzählung und die makellos fein gearbeitete Bildoberfläche ohne Spuren der Pinselführung (»finish«) niedriger - beides lange für das viktorianische Publikum die wichtigsten wertbildenden Eigenschaften eines Kunstwerks -, dafür den virtuosen Umgang mit Farbe und Form höher. Velázquez wurde so zum ersten Impressionisten erklärt, Whistler war sein legitimer Erbe. ${ }^{43}$ Die nächste Generation der britischen Kunstkritik wurde durch »Formalisten« wie Roger Fry (1866-1934) und Clive Bell (1881-1964) geprägt, doch waren Whistlers moderne Errungenschaften zu jenem Zeitpunkt längst von den aktuellen Abstraktionen der Avantgarde abgelöst worden. Er selbst gehörte zu diesem Zeitpunkt bereits zu den »alten Meistern«, und daran hatte Arrangement in Grey and Black: Portrait of the Painter's Mother wiederum keinen geringen Anteil. 
V.

\section{DER ANKAUF DES GEMÄLDES DURCH DEN FRANZÖSISCHEN}

STAAT

Whistlers Gemälde war seit seiner ersten Ausstellung 1872 in der Öffentlichkeit sehr präsent, nicht zuletzt durch Reproduktionen. Martha Tedeschi weist auf einen Holzschnitt in der Illustrated London News vom 8. Juni 1872 hin und auf professionelle Wiedergaben wie die Mezzotinto-Reproduktion von Richard Josey (1879), den Stich von Henri-Charles Guérard (1883) und die Lithographie von Thomas Way (1891). Auch Whistlers anlässlich seiner großen Retrospektive in der Londoner Goupil-Galerie 1892 produziertes Album mit fotografischen Reproduktionen einiger seiner Gemälde enthielt das Porträt seiner Mutter. ${ }^{44}$ Zuvor war das - zwischenzeitlich für zehn Jahre an die Kunsthandlung Henry Greaves \& Co. verpfändete - Bild auf zahlreichen Ausstellungen zu sehen gewesen und 1874 auch Bestandteil von Whistlers erster in Eigenregie veranstalteter Einzelausstellung in London. ${ }^{45}$ Anfang der 1880er Jahre wurde es in Philadelphia und New York gezeigt. 1883 kam es in den Pariser Salon, im darauf folgenden Jahr nach Dublin. Auf der III. Internationalen Kunstausstellung in München 1888 erhielt es eine Medaille zweiter Klasse. 1889 war das Gemälde in London, Amsterdam und Glasgow zu sehen.

Den größten Eindruck machte das Gemälde wohl in Paris. Ein Jahr nach dem Tod seiner Mutter stellte Whistler 1882 erstmalig seit 1873 in Paris, und erstmalig seit 1867 wieder im Salon aus. Das schlicht Portrait de ma mère betitelte Gemälde begleitete $1883 \mathrm{der}$ junge Walter Sickert als Kurier in den Pariser Salon. Der Kunstkritiker Théodore Duret (1838-1927) befand später in seiner WhistlerBiographie, dieses Bild habe den Künstler damals in Frankreich bekannt gemacht. Obwohl es nur mit einer Medaille dritter Klasse ausgezeichnet worden sei, habe es sich dabei um eine Form offizieller Anerkennung gehandelt, die dem Künstler in Großbritannien stets verwehrt geblieben sei. ${ }^{46}$ Diese Anerkennung hinderte zahlreiche Karikaturisten nicht daran, das Porträt aufs Korn zu nehmen und zu fragen, wieso der Künstler die alte Dame in einem Kohlensack habe posieren lassen, oder zu behaupten, das Bild stelle einen Protest gegen den Londoner Nebel dar. Die Kritik war 
gemischt und reichte von bissigen Kommentaren über das Porträt einer Dame nach ihrem Tod bis zu verständigen Bemerkungen zu den Farbharmonien, aber die Mehrheit der Meldungen in der Presse war positiv gestimmt. ${ }^{47}$. Auch in den folgenden Jahren fanden Whistlers Beiträge zum Salon und in den Pariser Galerien Georges Petit und Durand-Ruel mehr und mehr einflussreiche Fürsprecher in der Kunstkritik. ${ }^{48}$ Auf der Pariser Weltausstellung 1889 gewann sein Porträt von Lady Archibald Campbell (YMSM.242) eine Goldmedaille, und Whistler wurde zum Ritter (»Chevalier«) der Ehrenlegion ernannt. Als Außenseiter konnte er in Frankreich nun nicht mehr gelten, zumal er unter Kritikern und Dichtern wie Octave Mirbeau oder Stéphane Mallarmé gut vernetzt war - und diese wiederum beste Kontakte zur französischen Kulturverwaltung pflegten, etwa zu Henri Roujon (Directeur des Beaux-Arts) und Roger Marx (Inspecteur principal des musées départementaux und Herausgeber der Gazette des BeauxArts). Nachdem Whistlers Arrangement in Grey and Black, No. 2: Portrait of Thomas Carlyle (YMSM.137) im Frühjahr 1891 für 1000 Guineen von der Stadt Glasgow angekauft wurde, schlug sein Londoner Galerist, David Croal Thomson, über den Pariser Hauptsitz der Galerie Boussod, Valadon \& Cie vor, das Porträt der Mutter an das Musée du Luxembourg zu verkaufen. ${ }^{49}$ Das Museum war 1818 als Museum für lebende Künstler gegründet worden und wurde als "Vorzimmer des Louvre" angesehen. ${ }^{50}$ Whistler, seine Galerie und Mallarmé orchestrierten eine veritable Pressekampagne; die verantwortlichen Köpfe in der Kulturverwaltung wurden einer so intensiven wie erfolgreichen Lobbyarbeit ausgesetzt. ${ }^{51} \mathrm{Am}$ 27. November 1891 berichtete Le Figaro auf seiner Titelseite über den Ankauf des Gemäldes für das Musée du Luxembourg, das sich auch neben den alten Meistern im Louvre gut machen würde. 1925 zog das Porträt von Whistlers Mutter in den Louvre um, bevor es 1986 im Musée d'Orsay seinen heutigen Platz fand.

Die museale Kanonisierung seines Werkes in Frankreich hatte tiefgreifende Auswirkungen auf Whistlers Karriere. Zusammen mit seiner Frau Beatrice zog er 1892 von London nach Paris. Der Ankauf durch den französischen Staat wirkte als Erfolgskatalysator für die von David Croal Thomson 1892 in London orga-
47 Auszüge und Abbildungen in Margaret F. MacDonald, The Selling of Whistler's Mother, in: MacDonald (wie Anm. 4), 65-79, 69-70.

48 MacDonald (wie Anm. 47); zu Whistlers französischen Kunstkritikern siehe Joy Newton, Whistler's French Critics, in: Glazer et al. (wie Anm. 18), 81-93. 49 MacDonald (wie Anm. 47), 75; Pennell (wie Anm. 1), Bd. 2, 119.

50 Als Museum für lebende Künstler war das seit 1886 in der Orangerie unterge brachte, unter starkem Platzmangel leidende Musée du Luxembourg ein »musée de passage« - nach dem Tod eines Künstlers wurden dessen Werke in den Louvre gebracht. Vgl. Philippe de Chennevières u. a., Notice des peintures, sculptures et dessins de l'école moderne, exposés dans les galeries du Musée National du Luxembourg, Paris 1886, XIII. 51 MacDonald (wie Anm. 47), 75-76. 
nisierte Retrospektive von Whistlers malerischem Euvre. Während die Preise stiegen, konnte Whistler eine wachsende Zahl von vorwiegend amerikanischen Interessenten für Porträts verzeichnen. Vor allem amerikanische Museen zogen mit Ankäufen nach: Philadelphia (1895), Pittsburgh (1896), Boston (1896) und Chicago (1900). Da amerikanische Sammler vor allem in Paris Kunst kauften, wurde Whistler erst in Paris wieder zu einem amerikanischen Künstler. Sein Hauptwerk blieb indes in Frankreich - bis zu seiner USA-Tournee in den 1930er Jahren.

VI.

\section{Die TourneE DURCH DIE USA 1932-34}

Als Arrangement in Grey and Black: Portrait of the Painter's Mother in den Jahren 1932-34 in den USA auf Tournee ging, flackerte der Konflikt zwischen Form und Inhalt noch einmal auf. Kevin Sharp berichtet, wie das 1929 neu gegründete Museum of Modern Art in New York unter der grassierenden Weltwirtschaftskrise litt und der Gründungsdirektor Alfred H. Barr, Jr. (1902-1981) den Plan verfolgte, das Bild aus dem Louvre für eine Ausstellung moderner amerikanischer Kunst an das MoMa zu holen. ${ }^{52}$ Whistler war schließlich Amerikaner gewesen, auch wenn er seine gesamte Karriere in Paris und London verbracht hatte. Nach langwierigen diplomatischen Gesprächen schickte Frankreich das Meisterwerk als Zeichen der politischen Freundschaft über den Atlantik. Die Ausstellung wurde ein voller Erfolg. Zahlreiche amerikanische Museen fragten beim MoMa an, ob es möglich sei, Whistlers Mutter nach dem Ende der Ausstellung in New York zu ihnen zu schicken. Das Gemälde hatte in der öffentlichen Wahrnehmung bereits seinen alten Titel abgelegt und wurde nur noch als Mutter bezeichnet - und als Symbol aller amerikanischen Mütter verstanden und gefeiert. Der Louvre gab in einer für Frankreich politisch schwierigen Situation (das Land konnte Anleihen aus dem ersten Weltkrieg nicht pünktlich an die USA zurück zahlen) grünes Licht für eine Tournee, die bis zum Mai 1934 dauern sollte und Whistlers Porträt nach San Francisco, Los Angeles, St. Louis, Columbus (Ohio), Chicago, Cleveland, Kansas City, Baltimore, Toledo (Ohio), Dayton (Ohio) und Boston führen sollte, bevor es
52 Kevin Sharp, Pleasant dreams: Whistler's Mother on tour in America, 1932-4, in: MacDonald (wie Anm. 4), 81-99, 81-82. Die folgende Darstellung der Tournee beruht auf Sharps ausgezeichnetem Aufsatz. 
zum Abschluss noch einmal einige Tage im MoMa gezeigt wurde. Mehr als zwei Millionen Besucher konnten auf dieser Tournee einen Blick auf das Gemälde werfen. Es hatte in dieser Zeit durch eine sehr spezielle Rezeption eine ganz eigene Ikonographie der Mutterschaft zuerkannt bekommen, die mit einem Arrangement in Grau und Schwarz nicht mehr viel zu tun hatte. Dies ist umso erstaunlicher, als das Gemälde aus sich heraus eine solche Lesart nicht legitimiert, weil die Rolle der Mutter in ihm nicht erkennbar gemacht wird - der Titel »Die Witwe« wäre ebenso angemessen. Das Gemälde bot so den Anlass, unter dem Vorwand der Hochkultur ganz andere Inhalte zu feiern. Im Verlaufe der Tournee wurde es dabei immer wichtiger zu betonen, wie wertvoll das "\$1.000.00o-Bild« war. Zeitungen druckten Fotografien von bewaffneten Wachmännern mit ihren Schäferhunden vor der Mutter $\mathrm{ab}$, gewissermaßen eine ikonische Übersprunghandlung in Zeiten der Wirtschaftskrise, die den amerikanischen Müttern sagen sollte, sie könnten beruhigt schlafen gehen. ${ }^{53}$ Als die US-Postverwaltung 1834 zum bevorstehenden Muttertag eine Briefmarke herausgab, welche die unten angeschnittene und aus dem Bildkontext isolierte Figur aus Whistlers Portrait vor eine Blumenvase (mit Nelken) unter der Inschrift "In Memory and in Honor of the Mothers of America" platzierte (Abb. 7), war für Alfred H. Barr, Jr. das Fass übergelaufen. In einem offenen Brief, in der New York Times veröffentlicht, wies er auf Whistlers Intentionen hin, den eigentlichen Titel und die formalen Qualitäten des Gemäldes, ohne - wie Sharp betont - auf Mutterschaft, Versicherungswerte oder Schäferhunde einzugehen. ${ }^{54}$

Die Tournee ist ein frühes Beispiel dafür, wie aus einem Kunstwerk ein Medienereignis wird, in welchem das Objekt seine künstlerische Bedeutung fast vollständig verliert oder umgepolt bekommt. Whistlers Mother war zu einem Etikett geworden, das mit Whistler nur noch wenig zu tun hatte. Das Werk hatte sich von seinem Schöpfer emanzipiert. Es ist seitdem im kollektiven Gedächtnis der USA fest verankert, allerdings ohne seine formalästhetische Dimension, sondern als Ikone der eigenen Kultur, ${ }^{55}$ eine Art Berühmtheit, deren Bedeutung sich in ihrer Berühmtheit erschöpft, ohne dass dies als störend empfunden würde. Im letzten
53 Sharp (wie Anm. 52), 91. 54 Sharp (wie Anm. 52), 96, mit Hinweis auf Barrs Brief (Museum protests $>$ Whistler stamp<, The New York Times, 10. Mai 1934, 10).

55 Siehe hierzu Tedeschi (wie Anm. 44). Sie weist (a.a.O., 127-128) auf die Übereinstimmung der Pose der Mutter mit der Gestaltung von Porträtfotografien aus dem gleichen Zeitraum hin. Dies habe es dem Publikum erleichtert, sich das Bildnis von Whistlers Mutter als Teil einer visuellen Kultur anzueignen. 


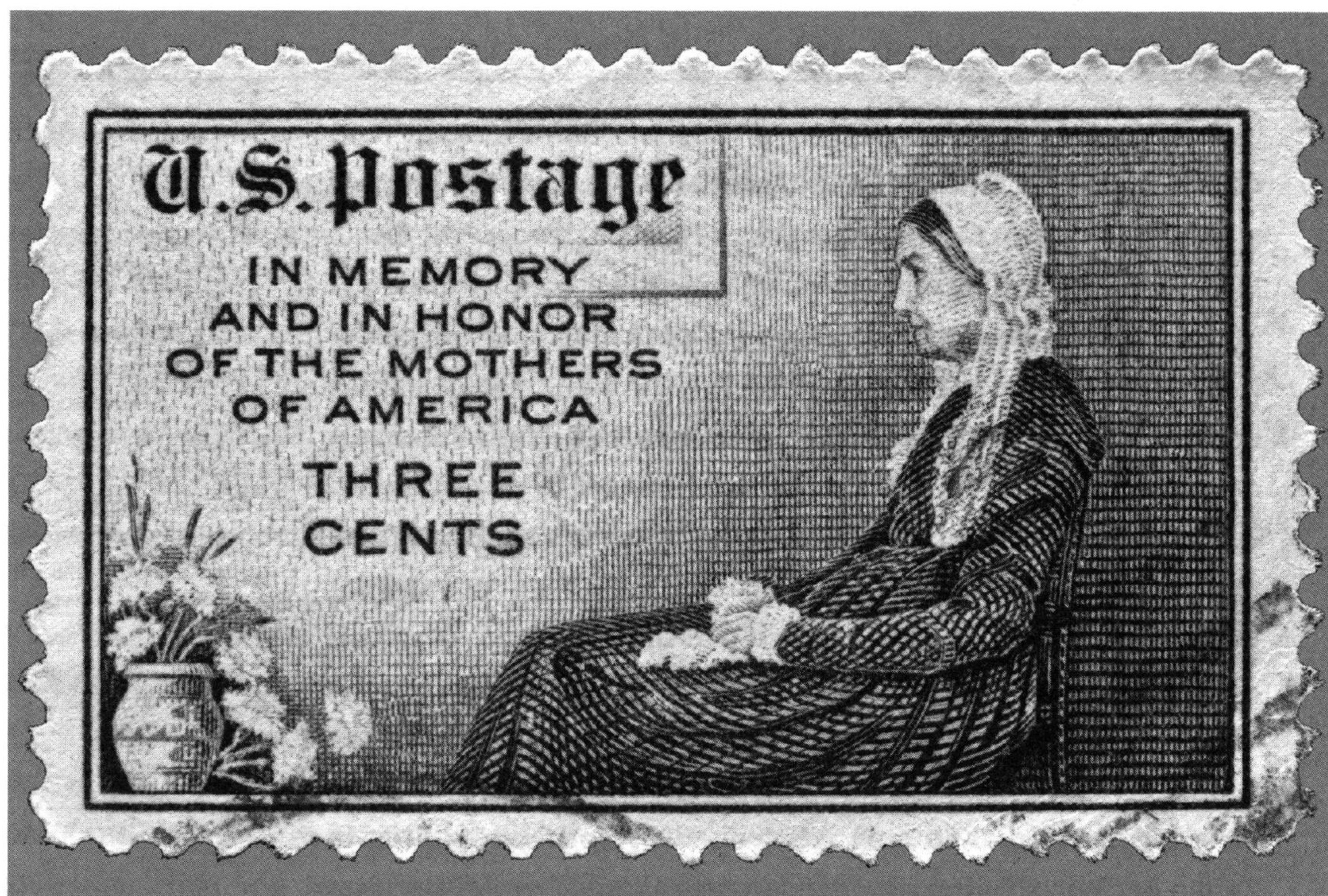

ABB. 7: BRIEFMARKE ZUM MUTTERTAG 1934, AUSGABEDATUM 2. MAI 1934, STAHLSTICHROTATIONSDRUCK, $21 \times 37$ MM, US-POSTVERWALTUNG, AUFLAGE 193.239.100 STÜCK

Jahr der Amerikatournee des Gemäldes schrieb der Jazzmusiker Cole Porter seinen Song You're the Top. Das Stück besteht im We56 Tedeschi (wie Anm. 44), sentlichen aus gegenseitigen Komplimenten eines Paares, die auf Referenzen an Prunkstücke der Kultur bestehen ("You're the Louvre Museum! - »You're Mickey Mouse!«), und so kommt die Mona Lisa genauso vor wie das amerikanische Meisterwerk schlechthin: "You're an O'Neill drama, you're Whistler's mama, you're Camembert!« Die Popkultur verleibte sich das Gemälde auch als Schablone für Cartoons, Plakate und die Werbung ein. Es dient, wie Tedeschi zusammenfasst, als Symbol für das Verhältnis der Amerikaner zu ihren Müttern (»love, respect, nostalgia, and occasional annoyance«), aber auch als Symbol für das Meisterwerk überhaupt. ${ }^{56}$

Der Blick auf seine Rezeptionsgeschichte erlaubt uns Arrangement in Grey and Black: Portrait of the Painter's Mother als ein Symptom der US-amerikanischen Kultur zu verstehen. Es ist nicht nur eine Antwort auf die Mona Lisa, weil es ein Amerikaner schaffte, sein Bild in den Louvre zu bringen, es ist in den USA auch 138. 
so bekannt wie die Mona Lisa, in der Tat eine "amerikanische Ikone«, wie der Untertitel des von MacDonald herausgegebenen Bandes feststellt. ${ }^{57}$ Als solche tritt sie auch im Film Bean - Der ultimative Katastrophenfilm von 1997 auf, in dem es bezeichnenderweise darum geht, dass das Gemälde an ein Museum in den USA verkauft wird - eine echte homecoming story. In den Kanon der 57 MacDonald (wie Anm. 4). $58 \mathrm{Zu}$ möglichen Gründen siehe Grischka Petri, Whistler and German Histories of Modern Painting: Another Case of Art and Art Critics, in: Glazer et al. (wie Anm. 18), $117-39$. deutschsprachigen Kunstgeschichte muss das Porträt erst noch aufgenommen werden. ${ }^{58}$ Das Gemälde antwortet auf ganz unterschiedliche Fragestellungen: Aspekte der modernen Abstraktion, sozialgeschichtliche Inhalte, seine Schlüsselstellung im Rahmen einer Künstlerkarriere, seine Rolle als Begleitmusik einer Biographie, sein Beitrag zur Institutionengeschichte, seine Stellung in der Kunstkritik und seine Popularität, die es zu einer amerikanischen Ikone haben werden lassen - diese Anknüpfungspunkte lassen eine Vielzahl methodischer Ansätze nicht nur zu, sondern verlangen geradezu nach ihnen. Anna Matilda Whistler wird aber ungerührt dieser Diskussionen weiter einfach nur in ihrem Rahmen sitzen, painted just as nice as possible. 